Nota Científica

\title{
Melanophthalma Motschulsky, 1866 (Coleoptera: Latridiidae) como visitante floral de Annona cherimola Miller, 1768 (Magnoliales: Annonaceae) en Chile central
}

\author{
Melanophthalma Motschulsky, 1866 (Coleoptera: Latridiidae) as floral visitor of \\ Annona cherimola Miller, 1768 (Magnoliales: Annonaceae) in central Chile
}

\begin{abstract}
Álvaro Urbina' ${ }^{1}$ Víctor Vicencio² ${ }^{2}$ José I. Hormaza ${ }^{3}$, Sophia Tobar ${ }^{1}$, Luis O. Aguado ${ }^{4}$, Jorge Lora ${ }^{3}$, Camila García $^{1,2}{ }^{\mathbb{D}}$, John Labarca ${ }^{2}$ y Eduardo Gratacós ${ }^{1,2 *}$
\end{abstract}

${ }^{1}$ Pontificia Universidad Católica de Valparaíso, Av. Brasil 2950, Valparaíso, Chile. ${ }^{2}$ Centro Regional de Investigación e Innovación para la Sostenibilidad de la Agricultura y los Territorios Rurales, Ceres. ${ }^{3}$ Instituto de Hortofruticultura Subtropical y Mediterránea La Mayora (IHSM La Mayora-CSIC-UMA), Subtropical Fruit Crops Department, Málaga, 29750, España. ${ }^{4}$ Andrena Iniciativas y estudios Medio ambientales, Cl Gabilondo 16Bis, Valladolid, 47002, España. 渔*eduardo.gratacos@pucv.cl

\section{ZooBank: urn:lsid:zoobank.org:pub:E230BD36-CF57-4A38-8C21-F3AEDA03E699}

https: / / doi.org / 10.35249/ rche.47.2.21.16

Resumen. La polinización del chirimoyo (Annona cherimola Miller) es uno de los aspectos más críticos de este cultivo debido a la dicogamia protogínica. Estudios muestran que el chirimoyo es polinizado por insectos silvestres, principalmente coleópteros. Sin embargo, en países donde el chirimoyo no crece naturalmente la polinización natural es deficiente. Por lo tanto, se realiza polinización manual la cual representa hasta un $50 \%$ de los costos operacionales del cultivo. El principal objetivo de este estudio fue caracterizar los visitantes florales del chirimoyo en Chile central. Los muestreos se realizaron durante la floración del chirimoyo ocurrida en enero y febrero de 2018, en tres huertos comerciales manejados con poda baja y una cubierta de herbáceas en la comuna de Quillota, Región de Valparaíso, Chile central. Se encontraron adultos del género Melanophthalma Motschulsky dentro de las flores de chirimoyo. Varios de esos individuos portaban polen de dicha especie vegetal adherido a diversas estructuras de su cuerpo, lo que posiciona a especies de este género como posibles polinizadores del cultivo de chirimoyo en Chile central.

Palabras clave: Anonácea; cultivo de chirimoyo; insectos silvestres; polen de chirimoyo; visitantes florales.

Abstract. Cherimoya (Anonna cherimola Miller) pollination is one of the most critical aspects of this crop due to protogyny dichogamy. Studies show that cherimoya tree pollination is carry on by wild insects, mainly coleopterans. However, in countries that cherimoya is not native, natural pollination is deficient. Therefore, hand- pollination is carried out, which represent $50 \%$ of operational costs of cherimoya crop. The mainly aim of this study was to characterizer flower visitors of cherimoya in central Chile. The sampleds were made during cherimoya tree blooming time in January and February of 2018, in three orchards managed with lowed pruning and herbaceous cover in Quillota district, Valparaíso region, central Chile. Was found adults of Melanophthalma Montschulsky on cherimoya flowers. Several of these individuals carried pollen of this species attached to various structures of their body, which positions species of this genus as possible pollinators of this crop in central Chile.

Recibido 21 Enero 2021 / Aceptado 14 Mayo 2021 / Publicado online 31 Mayo 2021

Editor Responsable: José Mondaca E. 
Key words: Anonaceae; cherimoya crop; cherimoya pollen; flower visitors; wild insects.

Annona cherimola Miller, 1768 (Magnoliales: Annonaceae), conocida como chirimoyo, es una planta nativa de los valles interandinos de Perú y Ecuador (Popenoe 1921), aunque se discute su posible origen mesoamericano (Larranaga et al. 2017). La polinización es uno de los aspectos más críticos de este cultivo. Esto se debe al fenómeno de la dicogamia protoginia, proceso en que la parte femenina y masculina maduran a destiempo, impidiendo la autopolinización (De La Rocha 1967). Sin embargo, Farré et al. (1976) afirman que puede haber coincidencia en las fases femeninas y masculinas. Esto último dependería de las condiciones ambientales, ya que la receptividad estigmática de la flor del chirimoyo está modulada ambientalmente, acortada por las altas temperaturas y prolongada por la alta humedad (Lora et al. 2011).

Antecedentes en especies del mismo género han mostrado que, en Anonna sp. la cuaja producida por autopolinización es de 1,5\% (George et al. 1989). Por otro lado, las flores de chirimoyo en estado femenino tienen una forma angosta muy alargada, abriendo desde el ápice; lo que impide el ingreso de insectos grandes como Apis mellifera Linnaeus, dificultando la polinización mediante insectos manejados.

Estudios demuestran que los coleópteros son los principales polinizadores de especies del género Annona Linnaeus (Gazit et al.1982; George et al. 1989; Nagel et al. 1989; Nadel y Peña 1994; Jenkins et al. 2013), encontrándose una relación positiva entre la presencia de coleópteros en las flores y la cuaja de frutos de Anonna squamosa Linnaeus y Anonna cherimola en Israel (Gazit et al. 1982) y Annona sp. en Australia (George 1989). Además, un estudio realizado en Japón mostró que las flores de chirimoyo fueron visitadas por miembros de las clases Aracnida e Insecta. De esta última se registraron visitas de miembros de los órdenes Dermaptera, Hemiptera, Lepidoptera, Thysanoptera y Coleoptera, siendo especies de este último orden las más frecuentes, principalmente las pertenecientes a las familias Rhizophagidae, Staphylinidae, Latridiidae y Nitidulidae (Tsukada et al. 2005).

Respecto a estudios realizados en Chile, López y Rojas (1992) describieron la presencia de Colopterus sp. y Carpophilus hemipterus Linnaeus (Nitidulidae) dentro de las flores de chirimoyo, mientras que Lopez y Uquillas (1997) verificaron la capacidad polinizadora de C. hemipterus. Además, López y Uquillas (1997) reportaron la presencia de Aridius sp., Corticaria sp. y Melanophthalma sp (Latriidae) dentro de las flores de chirimoyo. Sin embargo, las poblaciones de estos insectos en los sistemas actuales de cultivo en Chile central son muy bajas para asegurar una buena fecundación de las flores (López y Uquillas 1997), por lo cual, el déficit en la polinización natural del chirimoyo en Chile se resuelve mediante la polinización manual, labor que representa aproximadamente un $50 \%$ de los costos operacionales del cultivo (Agüero 2000). Entonces, es necesario determinar que insectos silvestres polinizan el chirimoyo en Chile para, en base a ello, generar estrategias que mejoren la polinización natural de esta especie. Por lo tanto, el principal objetivo de este estudio es identificar a los visitantes florales del chirimoyo y determinar la frecuencia de sus visitas en huertos de chirimoyo en Chile central. Un segundo objetivo es comprobar si los visitantes florales presentan polen de chirimoyo adherido a sus cuerpos.

Se realizaron muestreos durante los meses de enero y febrero de 2018, en tres huertos

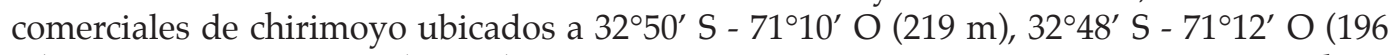
m) y $32^{\circ} 49^{\prime} \mathrm{S}-7^{\circ} 17^{\prime} \mathrm{O}$ (170 m) en la comuna de Quillota, Región de Valparaíso, Chile. Los tres huertos tenían árboles de chirimoyo variedad concha lisa, los cuales fueron plantados en la década de 1980, y se manejaron con una poda mínima, dejando que las plantas expresaran su hábito de crecimiento natural y se estableció una cubierta vegetal de plantas perennes compuesta principalmente por Cychorium intybus Linnaeus (Asterales: Asteraceae), Plantago lanceolata Linnaeus (Lamiales: Plantaginaceae), Coreopsis grandiflora 
Hogg ex Sweet (Asterales: Asteraceae), Mentha spicata Linnaeus (Lamiales: Lamiaceae) y Trifolium repens Linnaeus (Fabales: Fabaceae) con la finalidad de establecer un microhábitat diversificado y favorable para el desarrollo de las poblaciones de insectos.

En cada uno de los huertos se realizaron dos muestreos cada 14 días durante el periodo de floración del chirimoyo, sumando un total de 8 muestreos por predio. En cada muestreo se seleccionaron al azar nueve árboles por predio de los cuales se escogieron al azar flores en estado femenino (61BBCH, Cautín y Agustí 2005). Aunque no se fijó un número determinado de flores por cada árbol, entre los 9 árboles debían escogerse 100 flores en estado femenino. Por lo tanto, en cada muestreo se seleccionaron 300 flores sumando los tres predios, lo que da un total de 2400 flores ( 100 flores $\times 3$ huertos $=300$ flores $\times 8$ muestreos = 2400 flores). Cada observación se realizó entre las 9:00 am y las 12:00 pm (Nadel y Peña 1994), para lo cual se separaron cuidadosamente los tépalos y se observó la presencia de visitantes florales (Nagel et al. 1989), registrando la frecuencia de estos en las flores.

Las flores con coleópteros fueron recolectadas y almacenadas en un recipiente frío, para posteriormente ser trasladadas al laboratorio. Los coleópteros obtenidos se identificaron utilizando una lupa estereoscópica Zeiss Stemi modelo T4, de zum 4:1 y las claves taxonómicas de Majka et al. (2009) para la familia Latridiidae y Ewing y Cline (2005) para Nitidulidae. Los granos de polen presentes en los insectos se fotografiaron usando una cámara Imaging MicroPublisher 3.3 RTV conectada a un estereomicroscopio de fluorescencia (Olympus CX31). Las imágenes digitales se obtuvieron utilizando el software QCapturePro, versión 5.1.1.14 para Windows. Para determinar el origen del polen, estos fueron observados y comparados con las descripciones y fotografías de Lora et al. (2009) y Gan et al. (2015).

Aproximadamente el 2,4\% (58 de 2400 flores) de todas las flores observadas contenían coleópteros en su interior, y se capturó un total de 65 adultos. No se encontraron etapas inmaduras de los insectos dentro de las flores. Las familias recolectadas con mayor frecuencia correspondieron a Latridiidae con 52 individuos, Nitidulidae con 10 y Coccinellidae con 3 individuos. De la familia Nitidulidae, la única especie capturada dentro de las flores fue Carpophilus hemipterus Linnaeus. Estudios previos ya habían registrado a esta especie como polinizador del chirimoyo (Gazit et al. 1982; George et al. 1989; Nagel et al. 1989; López y Rojas 1992; López y Uquillas 1997). El género más frecuente fue Melanophthalma Motschulsky (Latridiidae), con 49 ejemplares capturados $(75,4 \%)$.

De los 49 individuos de Melanophthalma, 32 presentaron al menos un grano de polen. Sin embargo, solo se analizó el polen de 12 ejemplares que se observaron bajo la lupa detectando gran cantidad de granos adheridos a sus cuerpos registrando la presencia o ausencia de polen de chirimoyo. La forma globosa radiosimétrica del polen con un diámetro de alrededor de $40 \mu \mathrm{m}$ y la liberación de estos en tétradas, sugieren fuertemente que el polen transportado por los insectos pertenece a A. cherimola (Lora et al. 2009).

Aunque Melanophtalma fue registrado previamente por López y Uquillas (1997) como visitante floral del chirimoyo, el presente estudio destaca a especies de este género como el principal visitante floral de este cultivo en Chile central. Esto difiere con lo encontrado por López y Rojas (1992) ya que su estudio mostró que Carpophilus fue el género más frecuente que visitó las flores de chirimoyo. Asimismo, este estudio es el primer informe de la presencia de polen de chirimoyo en el cuerpo de miembros del género Melanophthalma encontrados en flores en estado femenino. Además, cabe aclarar que este estudio fue realizado durante una temporada de floración, por lo tanto, se hacen necesarios más años de muestreos para caracterizar de forma adecuada los visitantes florales del chirimoyo, ya que el ensamble de visitantes florales podría variar temporalmente.

Debido a las constantes visitas florales y a la presencia de polen de chirimoyo detectado en individuos de Melanophtalma spp. registrados en este estudio, junto al pequeño tamaño de los adultos (menos de 1,2 $\mathrm{mm}$ de longitud) que les permite ingresar al interior de las 
flores en estado femenino durante el día, y a la presencia de pilosidad corporal a la que se adhieren los granos de polen (Majka et al. 2009), se sugiere que este pequeño coleóptero es un potencial polinizador del cultivo del chirimoyo en Chile central. El estudio de estos insectos podría ayudar en el desarrollo de estrategias productivas enfocadas en la polinización natural de este frutal.

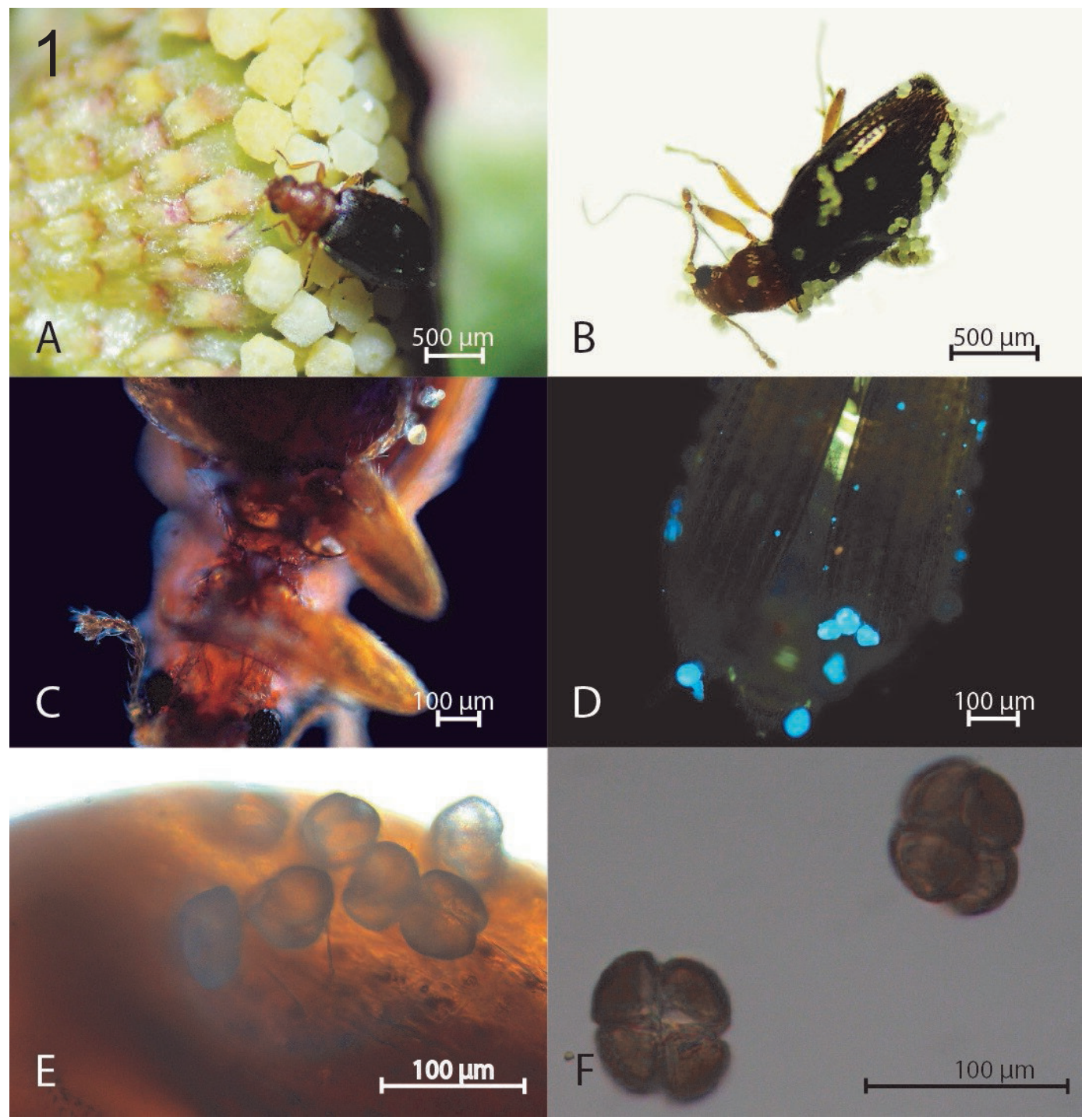

Figura 1. A. Adulto de Melanophthalma sp. dentro de flores de chirimoyo. B. Ejemplar cubierto de polen. C. Melanophthalma sp. observado bajo aumento con granos de polen adheridos a la parte anterior del abdomen. D. Élitros de Melanophthalma sp. observados bajo aumento y fluorescencia, con granos de polen adheridos a la pubescencia corporal. E. Detalle de la tétrada del polen de chirimoyo. F. Granos de polen con una disposición radio simétrica y forma globosa. / A. Adult of Melanophthalma sp. inside cheremolia flowers. B. Specimen covered in pollen. C. Melanophthalma sp. observed low magnification with pollen grains adhering to the anterior part of the abdomen. D. Elytra of Melanophthalma sp. observed under magnification and fluorescence, with pollen grains adhering to the body pubescence. E. Detail of the custard cheremolia tetrad. F. Pollen grains with a symmetrical radius arrangement and globose shape. 


\section{Agradecimientos}

Los autores agradecen el apoyo de los productores de chirimoya de la Región de Valparaíso: Juan Hargous, Luis Alvarado, Fernando Santa Cruz, Cesar Nagel, Dagmar Loos, Mónica Santelices, José Antonio Olaeta y Alberto Cortés Bustos de la Estación Experimental La Palma (PUCV) por facilitar sus huertos durante el desarrollo de los ensayos. Esta investigación fue financiada por el proyecto CONICYT REGIONAL / CERES / R16F10008 y ejecutado por el Centro Regional de Investigación e Innovación para la Sostenibilidad de la Agricultura y los Territorios Rurales (Ceres).

\section{Literatura Citada}

Agüero, G. (2000) Formulación y evaluación de un proyecto de plantación de chirimoyos en la zona de Quillota. Tesis Ingeniero Agrónomo. Universidad Católica de Valparaíso, Facultad de Agronomía. Quillota, Chile. 84 pp.

Cautín, R. y Agustí, M. (2005) Phenological growth stages of the cherimoya tree (Annona cherimola Mill.). Scientia Horticulturae, 105: 491-497.

De La Rocha, G. (1967) Cultivo de la chirimoya y resultados experimentales alcanzados. $2^{\mathrm{a}}$ ed. Perú, Ministerio de Agricultura, Centro Regional de Ayuda Técnica. 20 pp.

Ewing, C. y Cline, A. (2005) Key to adventive sap beetles (Coleoptera: Nitidulidae) in Hawaii, with notes on records and habits. The Coleopterists Bulletin, 59: 167-183.

Farré, J.M., Hermoso, J.M. y González, M.A. (1976) Ensayos sobre polinización, cuajado y crecimiento del fruto en chirimoya. Anales INIA/ Serie Producción Vegetal, 6(1976): 63-92.

Gan, Y., Liu, Y. y Xu, F. (2015) Pollen morphology of selected species from Annonaceae. Grana, 54: 271-281.

Gazit, S., Galon, I. y Podoler, H. (1982) The role of nitidulid beetles in natural pollination of Annona in Israel. Journal of the American Society for Horticultural Science, 107: 849-852.

George, A.P., Nissen, R.J., Ironside, D.A. y Anderson, P. (1989) Effects of nitidulid beetles on pollination and fruit set of Annona spp. hybrids. Scientia Horticulturae, 39: 289-299.

Jenkins, D.A., Cline, A., Irish, B. y Goenaga, R. (2013) Attraction of pollinators to Atemoya (Magnoliales: Annonaceae) in Puerto Rico: A synergistic approach using multiple nitidulid lures. Journal of Economic Entomology, 106(1): 305-310.

Larranaga, N., Albertazzi, F.J., Fontecha, G., Palmieri, M., Rainer, H., van Zonneveld, M. y Hormaza, J.I. (2017) A Mesoamerican origin of cherimoya (Annona cherimola Mill). Implications for the conservation of plant genetic resources. Molecular Ecology, 26(16): 4116-4130. https: / / doi.org/10.1111/ mec.14157

López, E. y Rojas, R. (1992) Artrópodos asociados a la floración del chirimoyo (Annona cherimola Mill.) en la localidad de Quillota, Quinta Región, Chile. Acta Entomológica Chilena, 17: 101-106.

López, E y Uquillas, C. (1997) Carpophilus hemipterus (Coleoptera: Nitidulidae) como agente polinizante de chirimoyo (Annona cherimola Mill.) bajo condiciones controladas. Acta Entomológica Chilena, 21: 88-89.

Lora,J., Testillano,P., Risueño,M.C.,Hormaza,J.I.yHerrero,M.(2009) Pollen development in Annona cherimola Mill. (Annonaceae). Implications for the evolution of aggregated pollen. BMC Plant Biology, 9: 129.

Lora, J., Herrero, M. y Hormaza, J. (2011) Stigmatic receptivity in a dichogamous earlydivergent angiosperm species, Annona cherimola (Annonaceae): Influence of temperature and humidity. American Journal of Botany, 98(2): 265-274.

Majka, C., Langor, D. y Ruckler, W. (2009) Latridiidae (Coleoptera) of Atlantic Canada: new records, keys to identification, new synonyms, distribution, and zoogeography. Canadian Entomologist, 141: 317-370. 
Nadel, H. y Peña, J. (1994) Identity, behavior, and efficacy of nitidulid beetles (Coleoptera: Nitidulidae) pollinating commercial Annona species in Florida. Environmental Entomology, 23: 878-886.

Nagel, J., Peña, E. y Habeck, D. (1989) Insect pollination of Atemoya in Florida. Florida Entomologist, 72: 207-211.

Popenoe, W. (1921) The native home of the cherimoya. Journal of Heredity, 12: 331-336.

Tsukada, M., Higuchi, H., Furukawa, T. y Yoshira, A. (2005) Flower visitors to cherimoya, Annona cherimola (Magnoliales: Annonaceae) in Japan. Applied Entomology and Zoology, 40: 317-324. 